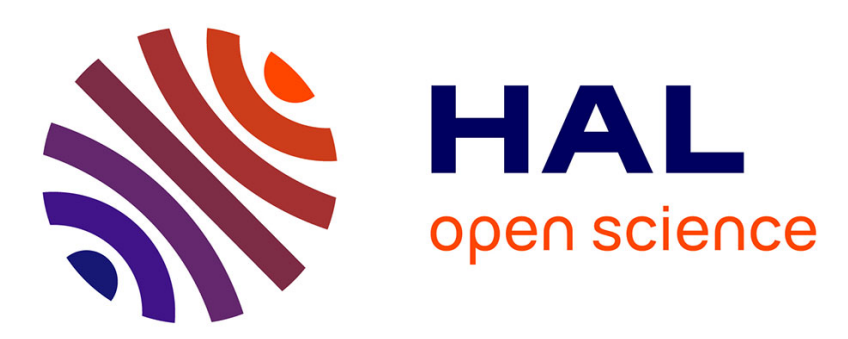

\title{
Full particle simulations of short large-amplitude magnetric structures (SLAMS) in quasi-parallel shocks.
}

K. Tsubouchi, Bertrand Lembège

\section{To cite this version:}

K. Tsubouchi, Bertrand Lembège. Full particle simulations of short large-amplitude magnetric structures (SLAMS) in quasi-parallel shocks.. Journal of Geophysical Research Space Physics, 2004, 109 (A2), pp.A02114. 10.1029/2003JA010014 . hal-00154729

\section{HAL Id: hal-00154729 \\ https://hal.science/hal-00154729}

Submitted on 23 Jan 2016

HAL is a multi-disciplinary open access archive for the deposit and dissemination of scientific research documents, whether they are published or not. The documents may come from teaching and research institutions in France or abroad, or from public or private research centers.
L'archive ouverte pluridisciplinaire HAL, est destinée au dépôt et à la diffusion de documents scientifiques de niveau recherche, publiés ou non, émanant des établissements d'enseignement et de recherche français ou étrangers, des laboratoires publics ou privés. 


\title{
Full particle simulations of short large-amplitude magnetic structures (SLAMS) in quasi-parallel shocks
}

\author{
K. Tsubouchi ${ }^{1}$ and B. Lembège \\ CETP/IPSL/CNRS, Velizy, France \\ Received 30 April 2003; revised 28 November 2003; accepted 4 December 2003; published 21 February 2004.
}

[1] Dynamics of SLAMS (short large-amplitude magnetic structures) is investigated by the use of one-dimensional, full particle electromagnetic simulations. As previous hybrid simulations and analysis of experimental observations suggested, present results confirm that the SLAMS patterns result from the steepening of long wavelength magnetosonic waves which are excited by diffuse ions (representing the field-aligned reflected ion beam) interacting with the upstream ambient plasma. Five successive phases have been identified in the SLAMS dynamics: ULF wave growth and symmetric, asymmetric, spiky, and late SLAMS. The present accessibility to high-resolution (electron) scales leads to the following new features: (1) the leading edge of the SLAMS steepens over a spatial scale from which a large-amplitude whistler precursor is emitted; (2) this whistler departs from the SLAMS edge and behaves as a new shock front; (3) the spiky SLAMS phase is characterized by the build-up of a strong spiky electrostatic field (its width is about 0.5 ion inertial length) within the whistler precursor and is intermittent with a lifetime less than one inverse ion gyroperiod; (4) the new shock front suffers a local self-reformation typical of a quasi-perpendicular shock in supercritical regime during the late-SLAMS phase. The features of the spiky SLAMS phase can be used as a typical signature in the time history of the SLAMS dynamics. Spatial/time scales of SLAMS have been measured throughout the different phases and are found in good agreement with results issued from previous hybrid simulations and with experimental measurements made by AMPTE UKS/IRM satellites; these are also compared with recent results from Cluster-2 space mission. INDEX TERMS: 7843 Space Plasma Physics: Numerical simulation studies; 7851 Space Plasma Physics: Shock waves; 2154 Interplanetary Physics: Planetary bow shocks; 2139 Interplanetary Physics: Interplanetary shocks; KEYWORDS: quasi-parallel shock, short large-amplitude magnetic structures, full particle simulation, bow shock, self-reformation

Citation: Tsubouchi, K., and B. Lembège (2004), Full particle simulations of short large-amplitude magnetic structures (SLAMS) in quasi-parallel shocks, J. Geophys. Res., 109, A02114, doi:10.1029/2003JA010014.

\section{Introduction}

[2] Short large-amplitude magnetic structures (so-called SLAMS) are one of the prominent features observed in the upstream region of a quasi-parallel shock. Schwartz et al. [1992] showed a series of SLAMS signatures identified from the data of AMPTE UKS/IRM satellites in the vicinity of the Earth's quasi-parallel bow shock. Mann et al. [1994] investigated the detailed statistics on the SLAMS-related quantities by using the same data interval of Schwartz et al. [1992]. In the work of Schwartz et al. [1992], the main characteristics of observed SLAMS were defined as (1) short duration in the range 5-20 s, (2) large amplitude of magnetic field amplitude $|B|$ by the factor of 2 or more above the background field, and (3) well-defined single

\footnotetext{
${ }^{1}$ Now at Radio Science Center for Space and Atmosphere, Kyoto University, Uji, Kyoto, Japan.

Copyright 2004 by the American Geophysical Union. 0148-0227/04/2003JA010014
}

monolithic pattern in $|B|$. Detailed analysis further showed that the propagation speed of SLAMS in the nominal rest frame of the shock decreases with increasing $|B|$ [Schwartz et al., 1992], and the direction of the magnetic field within SLAMS points more perpendicular to the nominal shock normal than in the undisturbed background [Mann et al., 1994]. In the quasi-parallel regime, ultra low-frequency (ULF) magnetosonic waves can be produced by the beam instabilities associated with the reflected ions backstreaming from the shock along the upstream magnetic field. Giacalone et al. [1993] suggested from the same AMPTE data that SLAMS are the result of the steepening of such ULF waves as they pass through an energetic ion pressure gradient ahead of the shock front. Schwartz and Burgess [1991] argued the scenario that SLAMS originate directly from these upstream ULF waves and that a quasi-parallel shock transition can be regarded as a three-dimensional patchwork of ULF waves and SLAMS.

[3] We can further consider how ULF waves/SLAMS can be associated with the cyclic behavior of a quasi-parallel 
shock reformation [Burgess, 1989]. Among a number of numerical simulation studies which attempt to explain the reformation mechanism, Scholer [1993] and Giacalone et al. [1994] successfully retrieved magnetic structures very similar to the SLAMS which lead to reforming shocks (for other mechanisms, see the introduction of Scholer [1993]). According to Scholer [1993, hereinafter referred to as Paper 1], with a sufficiently oblique angle between the upstream magnetic field and the shock normal $\left(\theta_{B o}=30^{\circ}\right)$, a magnetosonic wave is excited by an ion/ion beam instability between the background plasma and diffuse backstreaming ions (representing the hot beam) with a spatial scale over 20 ion inertial lengths upstream of the shock ramp, much larger than the distance specularly reflected ions could reach. When the density gradient scale of the upstream diffuse ions is comparable to this ULF wavelength, the wave suffers a strong shrinking and steepening and becomes a pulse-like structure (associated with so-called SLAMS) as it is carried back toward the shock. Paper 1 concluded that upstream ULF waves and SLAMS are the same entity in different stages of their development and play a crucial role in reforming oblique quasi-parallel shock.

[4] In contrast with most previous studies based on the use of hybrid simulations (particle ions and massless fluid electrons) as in Paper 1 and in the work of Giacalone et al. [1994], full-particle simulations have been poorly used for analyzing the dynamics of quasi-parallel shocks and/or SLAMS formation. In the hybrid code, it is difficult to ensure the proper resolution for analyzing phenomena which have scales close to or less than ion inertial length and ion gyroperiod. In addition, the build-up of self-consistent space charge separation field cannot be established in hybrid simulations; instead, the electrostatic field results from imposing the quasi-neutrality condition. In Paper 1, emission of short wavelength whistler waves from the "old" shock ramp (when the magnetic pulse crashes into the shock) or from the steepened upstream edge is evidenced, but it was recognized that these whistler waves are not well resolved because of the poor spatial resolution. Therefore it is definitely important to analyze the quasiparallel shock and SLAMS dynamics under conditions where accessibility to the smallest physical scale (electron) is allowed in order to understand these time/space evolution more accurately. Using full particle code enables such analyses. In a previous full particle approach, Pantellini et al. [1992] have focused on the self reformation of the quasiparallel shocks based on a different process. A whistler wave train is emitted dispersively by the steepened shock ramp. This whistler precursor grows until it reaches a critical amplitude and a critical spatial extent so that a significant number of ions become trapped. Phase mixing thermalizes shortly the ions within the interaction region which becomes the part of the downstream region so that a new shock ramp transition builds up near the leading edge of the whistler wavetrain. However, at that time, no SLAMS pattern has been identified in this study. Among the various self-reformation processes proposed in the intrinsic dynamics of quasi-parallel shocks, only one clearly leads to the formation of monolithic B profiles approaching the characteristics of SLAMS [Scholer, 1993].

[5] Until now, no quantitative analysis has been explicitly focused on the detailed features/scales of the SLAMS during their time evolution from ULF growth, by the use of a full particle simulation. Giacalone et al. [1994] suggested from one-dimensional (1-D) hybrid simulations that "SLAMS" or SLAMS-like structures evolve very quickly and significantly different field signatures should be observed even by multi-spacecrafts which are separated each other only a few hundreds kilometers ( $\sim$ a few upstream ion inertial lengths). Recent Cluster-2 multi-spacecraft observations [Lucek et al., 2002] showed significant variations between the magnetic field magnitude signatures of the SLAMS measured by spacecrafts only $\approx 400-1000 \mathrm{~km}$ apart, although the overall size of the SLAMS exceeded this scale. These observations suggest that such differences are dominated by spatial variations and SLAMS are 3-D entities, although these might hide additional differences arising from SLAMS evolution with time. In the present study, we follow how SLAMS are growing with time and space via one-dimensional numerical simulations. In such a case, the effects due to the multidimensionality, such as wave refraction or filamentation [Dubouloz and Scholer, 1995] are ignored. However, two-dimensional simulations of Dubouloz and Scholer [1995] confirm quite well main results issued from one-dimensional simulations [Dubouloz and Scholer, 1993], i.e., backstreaming ion beams initially generate ULF waves in the RHR (right-hand resonant) mode. These waves are convected downstream in regions of increasing beam density and reach large amplitudes so that they scatter the ion beams forming clumps containing a significant fraction of ions with velocities antiparallel to the beam bulk velocity. This enables the destabilization of the LHR mode [Gary et al., 1984]. The final result of this process is the formation of enhanced magnetic structures which are very similar to the pulsations observed upstream of the Earth's bow shock. Twodimensional effects only enable a more realistic investigation of the pulsation properties which substantiates in a quantitative way the idea proposed by Schwartz and Burgess [1991] that quasi-parallel shocks may consist of a multidimensional patchwork of such structures.

[6] These previous results and Paper 1 confirm that onedimensional simulations are appropriate to analyze the basic processes of SLAMS formation from the upstream ULF waves in the presence of strong diffuse ion gradient. In the present study, we use a PIC (particle-in-cell) code which treats both electrons and ions as individual particles. Our purposes are (1) to identify the detailed time/spatial scales of SLAMS with high resolution at different stages of its evolution within a full self-consistent approach including both electron and ion scales, (2) to compare present results with those of Paper 1 for validation, (3) to identify new features of SLAMS related to the use of a PIC code, (4) to confirm that the properties of the obtained SLAMS are similar to those observed by spacecrafts, and (5) to provide estimates of Cluster-2 spacecrafts separation for analyzing SLAMS pattern at different times of its development. Full dynamics of the quasi-parallel shock and associated cyclic reformation processes over very long time scales will be analyzed in a further paper.

\section{Conditions of Numerical Simulations}

[7] Initial and boundary conditions used in the present 1-D electromagnetic full particle simulations are identical to 
Table 1. Upstream Plasma Parameters Defined for Full Particle Simulations

\begin{tabular}{lcc}
\hline & Electrons & Ions \\
\hline$\widetilde{v}_{t h}$ & 0.75 & 0.1 \\
$\widetilde{\lambda}_{D}$ & 0.75 & 0.75 \\
$\widetilde{\rho}_{c}$ & 3 & 21.21 \\
$\widetilde{c} / \widetilde{\omega}_{p}$ & 6 & 42.43 \\
$\widetilde{\omega}_{c}$ & 0.25 & 0.005 \\
$\widetilde{\omega}_{p}$ & 1 & 0.141 \\
$\widetilde{\tau}_{c}$ & 25 & 1256 \\
$\beta$ & 0.5 & 0.5 \\
\hline
\end{tabular}

those already explained by Lembège and Dawson [1987]. Briefly, the shock is propagating along $x$ axis, and a static magnetic field $B_{o}$ is applied within the plane $(x, z)$ so that its propagation direction is defined by the angle $\theta_{o}=\left(\overrightarrow{B_{o}}, \vec{x}\right)=$ $30^{\circ}$. All dimensionless quantities are indicated by a tilde " $\sim$ " and are normalized as follows. The spatial coordinate is $\widetilde{x}=$ $x / \Delta$; velocity $\widetilde{v}=v / \omega_{p e} \Delta$; momentum of species $\gamma, \widetilde{p}_{\gamma}=$ $p_{\gamma} / m_{e} \omega_{p e} \Delta$; electric field $\widetilde{E}=q E / m_{e} \omega_{p e}^{2} \Delta$; magnetic field $\widetilde{B}=q B / m_{e} \omega_{p e}^{2} \Delta ;$ time $\widetilde{t}=\omega_{p e} t$. The parameters $\Delta, \omega_{p e}, m_{e}, q$ and $n_{o}$ are the numerical grid spacing, the electron plasma frequency at $\widetilde{t}=0$, the electron mass, the electric charge, and the particle density at $\mathrm{t}=0$, respectively.

[8] The basic parameters are plasma box size length $\widetilde{L}_{x}=$ $30720 \Delta$ (covering $724 \widetilde{c} / \widetilde{\omega_{p i}}$ ); velocity of light $\widetilde{c}=6$, mass ratio $m_{e} / m_{i}=0.02$. At $\widetilde{t}=0$, the particle density is $n_{e}=n_{i}=$ 20 at each grid point and the temperature ratio is $T_{e} / T_{i}=1$, the magnetic field $\left|\widetilde{B}_{o}\right|=1.5$.

[9] For these initial conditions, the plasma parameters are summarized in Table 1 for both electrons and ions. The size of the simulation box is large enough to allow the occurrence of upstream ULF waves. The shock is propagating in a supercritical regime $\left(M_{A}=5.8\right)$, where $M_{A}=\widetilde{v}_{\text {shock }} / \widetilde{v}_{A}$ has been determined in the upstream plasma (i.e., simulation) frame; the Alfvén velocity $\widetilde{v}_{A}$ is equal to 0.21 . Let us precise that the shock front velocity has been measured just before any ULF wave and subsequent SLAMS build up. As shown in section 3 , this "local time" velocity value $5.8 \widetilde{v}_{A}$ is larger

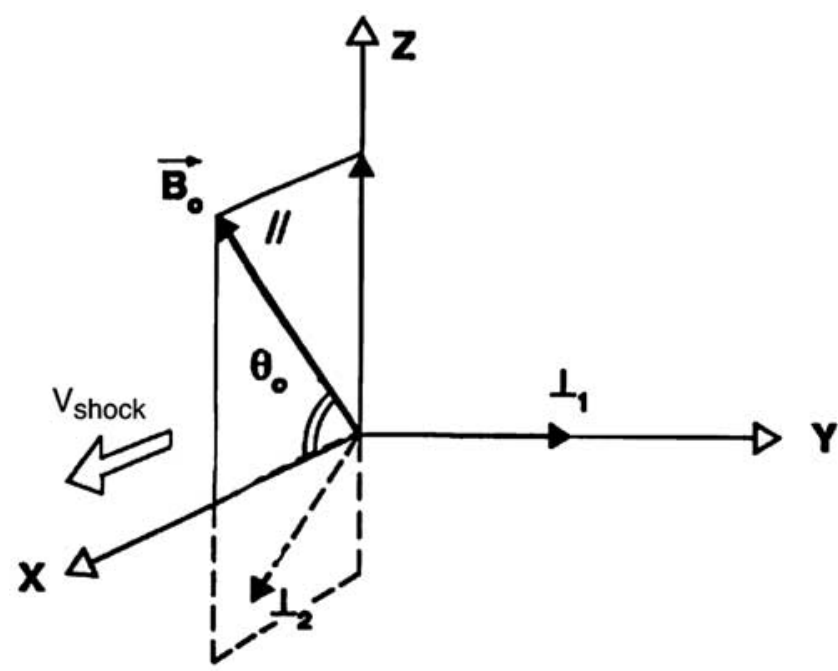

Figure 1. Reference frame used in the 1D simulation for the quasi-parallel shock; $\widetilde{B}_{o}$ is the static magnetic field. The shock is propagating along $\mathrm{x}$-axis. than the SLAMS velocity itself; let us note that the velocity of shock front is strongly varying in time because of the associated self-reformation process.

[10] The reference set is plotted in Figure 1. The size of the box allows to follow the shock and particle dynamics over a time $\widetilde{t} \approx 42 \widetilde{\omega}_{c i}^{-1}$, where $\widetilde{\omega}_{c i}$ is the upstream ion gyrofrequency. In order to initiate the shock, we have used the magnetic piston method (applied current pulse) which reveals to be quite efficient and rapid [Lembège and Dawson, 1987; Lembège, 2003]. After a transitory time about $\widetilde{t}=4 \widetilde{\omega}_{c i}^{-1}$, a shock front is formed and an important percentage of ions are reflected and backstream along the upstream magnetic field.

\section{Simulation Results}

\subsection{Time Evolution of SLAMS}

[11] Figure 2 shows the time stackplots of the magnetic field amplitude $|\widetilde{B}|$ within a selected time range of the entire simulation $\left(19.2 \widetilde{\omega}_{c i}^{-1}<\widetilde{t}<28.8 \widetilde{\omega}_{c i}^{-1}\right)$. The spatial extent $\left(117.8 \widetilde{c} / \widetilde{\omega}_{p i}\right)$ in the figure corresponds to an expanded and partial part of the whole simulation box. The time interval between two neighboring profiles is $0.24 \widetilde{\omega}_{c i}^{-1}$. At $\widetilde{t}=$ $19.2 \widetilde{\omega}_{c i}^{-1}$, the leading edge of the shock front is located at $\tilde{x} \sim 8820$ and propagates rightward. Within an upstream distance $\widetilde{L}=\widetilde{\lambda} / 2 \sim 35 \widetilde{c} / \widetilde{\omega}_{p i}$ (i.e., $9000<\widetilde{x}<10500$ ) from the shock front, the magnetic field is slightly larger than its background upstream value, which corresponds to the growth of a ULF wave (wavelength $\widetilde{\lambda}$ ) excited by the interaction between upstream ions and the reflected ions beam. Before $\widetilde{t} \sim 21.6 \widetilde{\omega}_{c i}^{-1}$, the propagation speed of the ULF wave is approximately $3 \widetilde{v}_{A}$ in the upstream direction.

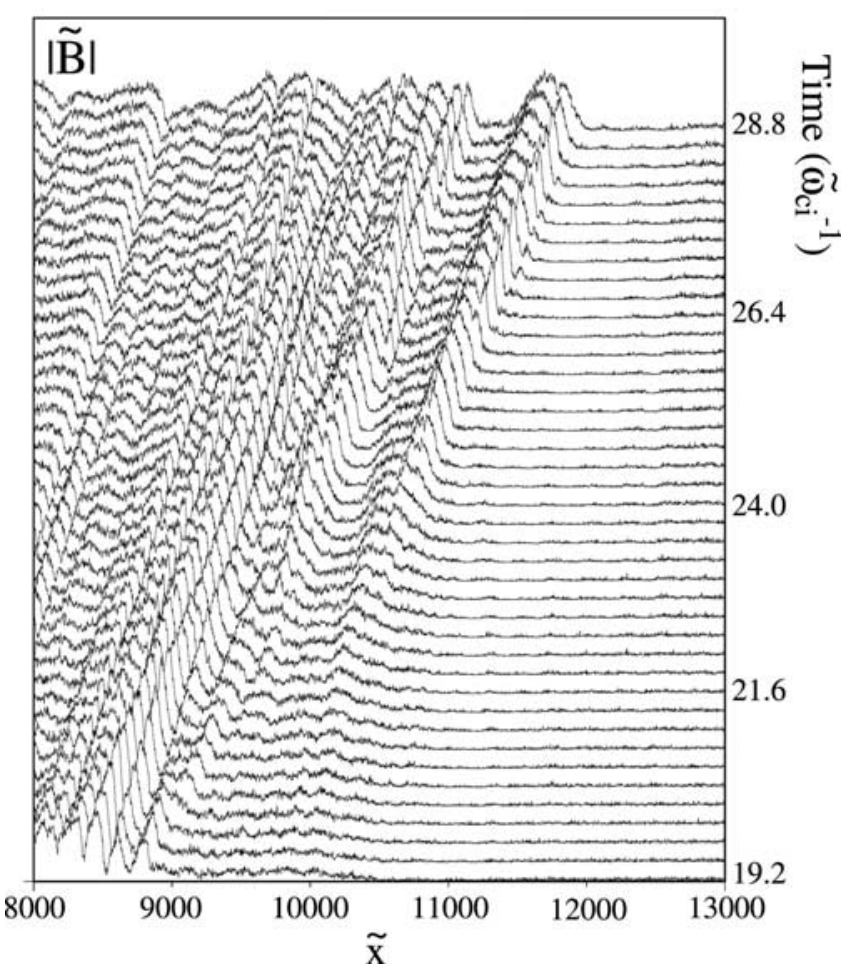

Figure 2. Time stackplot of the magnetic field amplitude profile $|\widetilde{B}|$ for $\theta_{B o}=30^{\circ}$ and $M_{A} \sim 5.8$. The time interval between two successive profiles is $0.24 \widetilde{\omega}_{c i}^{-1}$. 
This means the ULF wave is carried back relatively toward the shock front by an incoming solar wind with velocity $2.8 \widetilde{v}_{A}$ in the shock-rest frame.

[12] After $\widetilde{t} \sim 21.6 \widetilde{\omega}_{c i}^{-1}$, this ULF wave rapidly begins shrinking and a distinct pulse-like structure emerges from its background. Within a time range $\sim 2-3 \widetilde{\omega}_{c i}^{-1}$, its spatial scale is reduced to less than $20 \widetilde{c} / \widetilde{\omega}_{p i}$ and its magnitude is amplified from 3 to 5 times its upstream $\left|\widetilde{B}_{o}\right|$ value. This pulse-like pattern is clearly detached from the old shock front and shows a monolithic profile which is one clear feature of SLAMS [Schwartz et al., 1992]. As the SLAMS further approaches the shock, its speed is decelerated and becomes almost phase-standing in the shock rest frame. The leading edge of the SLAMS continues steepening and is finally acting as a new shock front around $\tilde{t} \sim 26.4 \widetilde{\omega}_{c i}^{-1}$. At this stage, one can consider that one cycle of the quasiparallel shock reformation is completed. These features are consistent with the hybrid simulations of Paper 1 where the shock reformation is based on the growth, the shrinking and the steepening of upstream ULF waves.

[13] From Figure 2, we can evidence the rapid evolution of the SLAMS into five successive phases. The first four phases are detailed in Figures 3 to 5. Spatial profiles of $|\widetilde{B}|$, transverse components of the magnetic field $\widetilde{B}_{y, z}$, the electrostatic field $E_{l x}$, and the ion phase space $\left(\widetilde{v}_{x, i}-\widetilde{x}\right)$ are plotted in Figures $3 \mathrm{a}$ to $3 \mathrm{~d}$. Corresponding space profiles of the rotation angles $\left(\theta_{B}\right.$ and $\left.\phi_{B}\right)$ of the magnetic field are plotted in Figure 4, where angles $\theta_{B}$ and $\phi_{B}$ are defined within and outside the coplanarity plane (containing the upstream field $\widetilde{B}_{o}$ and the normal to the shock front). The fifth phase is described in Figures 6 to 7.

\subsection{ULF Wave Growth}

[14] At this stage, a large percentage of upstream ions have been reflected against the shock front, stream back along the upstream magnetic field lines, and start building up a ULF wave (upstream ion/ion beam instability). This phase corresponds to the linear wave growth (Figure 3a). In the $|\widetilde{B}|$ profile, the wave scale length extends over $\widetilde{L} \sim 35 \widetilde{c} / \widetilde{\omega}_{p i}$ from the shock front with $|\Delta \widetilde{B}| /\left|\widetilde{B}_{o}\right| \approx 0.86$. The frequency of the ULF wave of which leading edge propagates at velocity $\approx 3 \widetilde{v}_{A}$ is estimated to $\widetilde{\omega} \sim 0.5 \widetilde{\omega}_{c i}$. In particular, the spatial length scale is much larger than the distance specularly reflected ions can reach and is almost equivalent to the scale of diffuse ion density gradient (no shown herein), in a good agreement with results of Paper 1.

[15] In the present case, $\left(\widetilde{v}_{x, i}-\widetilde{x}\right)$ ion phase space in Figure 3 a shows that ions are only partially diffuse in the upstream region away from the ULF wave $\widetilde{x}>11000)$ and are strongly diffuse at the location where the ULF wave becomes noticeable $(9000<\tilde{x}<10500)$. The mechanisms responsible for this ion diffusion are presently under active investigation. Moreover, the phase relation deduced from $\widetilde{B}_{y}$ and $\widetilde{B}_{z}$ profiles shows that the ULF wave is right-hand polarized in the upstream rest frame (not shown here). The associated space charge effects are still relatively weak so that the amplitude of the electrostatic field is almost negligible at that time (Figure 3a).

\subsection{Symmetric SLAMS}

[16] At $\widetilde{t}=23.6 \widetilde{\omega}_{c i}^{-1}$, a distinct SLAMS starts building up between $\widetilde{x} \sim 10300$ and $\widetilde{x} \sim 10900$ (Figure 3b). We name this phase "symmetric SLAMS" from the "monolithic" spatial signature of $|\widetilde{B}|$. Within $4 \widetilde{\omega}_{c i}{ }^{-1}$ after the "ULF wave growth" phase, the peak amplitude increases to $|\Delta \widetilde{B}| /\left|\widetilde{B}_{o}\right| \approx 2.66$. This amplification process is simultaneously accompanied by a shrinking of the SLAMS width from $35 \widetilde{c} / \widetilde{\omega}_{p i}$ to $14 \widetilde{c} / \widetilde{\omega}_{p i}$. Correspondingly, background ions suffer a strong bulk motion (compression) at the location where the SLAMS builds up. The magnetic field polarization in the SLAMS is unchanged (right-hand in the upstream rest frame). As a consequence of the shrinking, characteristic spatial widths may now be defined respectively in the ramp measured at the leading edge $\left(254 \Delta \approx 6 \widetilde{c} / \widetilde{\omega}_{p i}\right)$ and at the trailing edge $(150 \Delta \approx$ $\left.3.54 \widetilde{c} / \widetilde{\omega}_{p i}\right)$. The velocity measured from the leading edge motion is $4.47 \widetilde{v}_{A}$. At this stage, a macroscopic electrostatic field $\widetilde{E}_{l x}$ starts building up (Figure $3 \mathrm{~b}$ ). Through the SLAMS, the $\widetilde{B}$ field suffers some noticeable rotation both in $\theta_{B}$ and in $\phi_{B}$ angles; these angle variations present a symmetry at the leading and the trailing edges (Figure 4b). Moreover, noticeable deviations are evidenced through the old shock ramp "O." In summary, a net separation appears both in the enhancements of the $\widetilde{B}$ field and in the variations of the magnetic field direction between the newly born SLAMS and the old shock front "O."

\subsection{Asymmetric SLAMS}

[17] Transition from "symmetric SLAMS" phase to the next phase "asymmetric SLAMS" is relatively quick occurring within a time less than $2 \widetilde{\omega}_{c i}^{-1}$. Spatial profiles at $\widetilde{t}=25.2 \widetilde{\omega}_{c i}^{-1}$ are plotted in Figure $3 \mathrm{c}$. The magnetic field amplitude is further amplified at the leading edge of the SLAMS $\left(|\Delta \widetilde{B}| /\left|\widetilde{B}_{o}\right| \approx 4.4\right)$ in contrast with little variation in the trailing region $\left(|\Delta \widetilde{B}| /\left|\widetilde{B}_{o}\right| \approx 1.6\right)$ which leads to the formation of a plateau. Accordingly, though the overall polarization still stays right-hand as in previous phases, $B_{y}$ and $B_{z}$ components become more linearly correlated. At the leading edge $(\tilde{x} \sim 11000)$, the magnetic field is not only amplified but becomes also strongly steepened. This steepening is mainly carried by electron current. At this stage, the spatial width of the SLAMS is almost unchanged $\left(\sim 14.2 \widetilde{c} / \widetilde{\omega}_{p i}\right)$. However, the width of the leading edge ramp strongly reduces to $2.47 \widetilde{c} / \widetilde{\omega}_{p i}$ in contrast with the width of the trailing edge $\operatorname{ramp}\left(5.11 \widetilde{c} / \widetilde{\omega}_{p i}\right)$. Steepening becomes the dominant process, while the wave shrinking process almost stops. Since electrons and ions behave quite differently at the steepened leading edge, a noticeable local electrostatic field $\widetilde{E}_{l x}$ builds up there (Figure $3 \mathrm{c}$ ). A more complete analysis allows to show that the leading edge starts acting as a new local quasi-perpendicular shock front ("N") characterized as follows: (1) a certain percentage of the background upstream ions start reflected, initiate a characteristic trapping loop (Figure 3c) and suffer a local $\vec{E} \times \vec{B}$ acceleration similar to that usually observed in quasi-perpendicular shock (not shown herein), and (2) the $B$ field is strongly rotating within and outside the coplanarity plane to reach angles value $\theta_{B}=80$ and $\phi_{B}=-50$ (Figure $4 \mathrm{c}$ ). At this stage, $\theta_{B}$ is always positive at the leading edge. These values are in a good agreement with angle variations through an oblique quasiperpendicular shocks as observed by Savoini and Lembège 

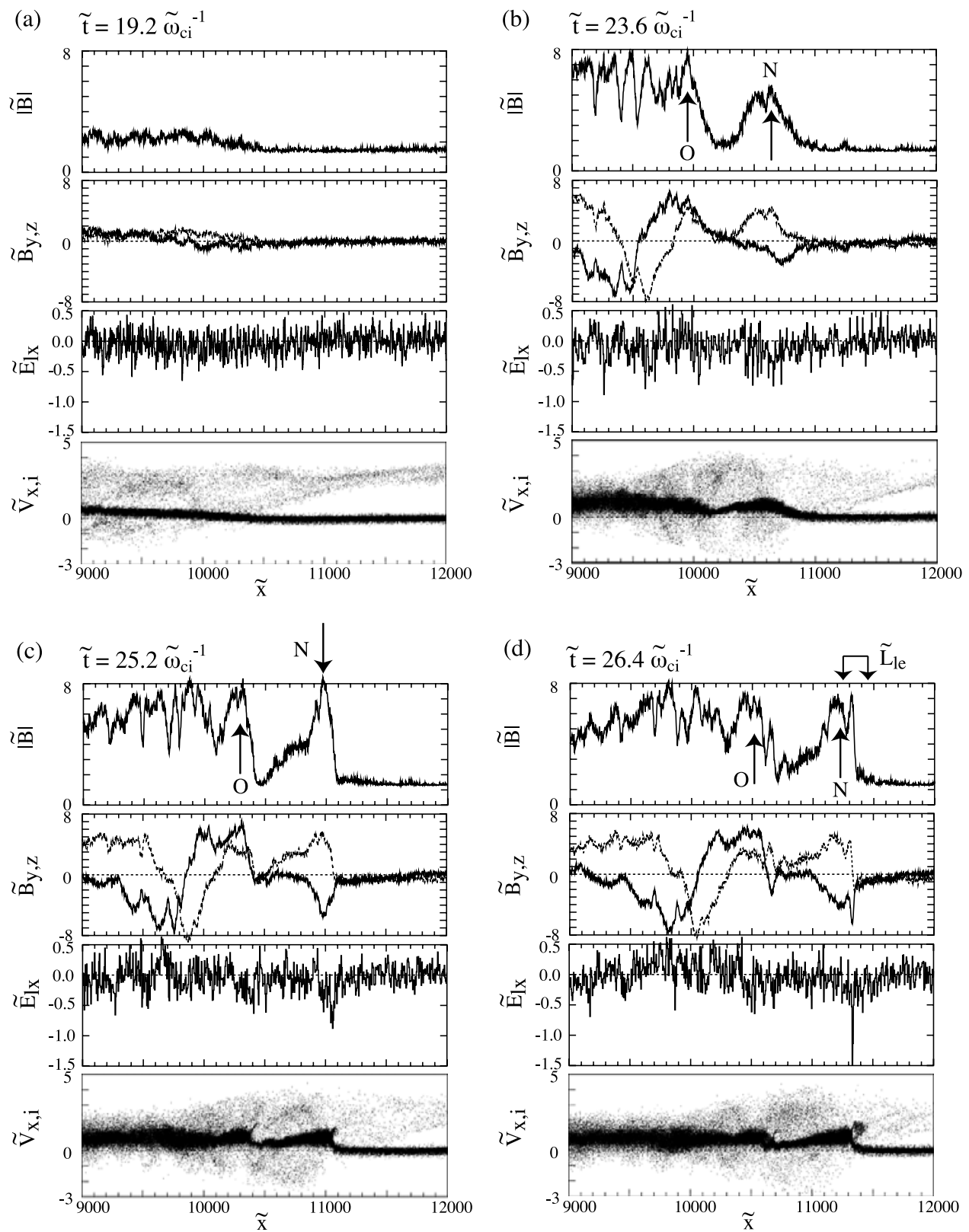

Figure 3. Main phases of the SLAMS dynamics showing the spatial profiles of the $|\widetilde{B}|$ amplitude, transverse components of the magnetic field $\widetilde{B}_{y}$ (solid line) and $\widetilde{B}_{z}$ (dashed line; $\widetilde{B}_{z 0}$ removed), the electrostatic field $\widetilde{E}_{l x}$, and the ion phase space $\left(\widetilde{v}_{x, i}, \widetilde{x}\right)$. Figures 3 a to $3 \mathrm{~d}$ correspond to the "ULF growth" phase at $\widetilde{t}=19.2 \widetilde{\omega}_{c i}^{-1}$ (i.e., at $3840 \widetilde{\omega}_{p e}^{-1}$ ), the "Symmetric SLAMS" phase at $\widetilde{t}=23.6 \widetilde{\omega}_{c i}^{-1}$ (i.e., at $4728 \widetilde{\omega}_{p e}^{-1}$ ), the "Asymmetric SLAMS" phase at $\widetilde{t}=25.2 \widetilde{\omega}_{c i}^{-1}$ (i.e., at $5040 \widetilde{\omega}_{p e}^{-1}$ ), and to the "Spiky SLAMS" phase at $\widetilde{t}=26.4 \widetilde{\omega}_{c i}^{-1}$ (i.e., at $5280 \widetilde{\omega}_{p e}^{-1}$ ), respectively.

[1994]. This local rotation is the consequence of the SLAMS steepening which results in the linear polarization between $\widetilde{B}_{y}$ and $\widetilde{B}_{z}$ components at the new front "N." Although the $\widetilde{B}$ field amplitude strongly varies over the SLAMS extent, the angle $\theta_{B}$ stays almost constant around $75^{\circ}$. Moreover, let us note that background ions simultaneously start a local reflection around the old shock front "O" which behaves as a local quasi-perpendicular shock too (Figures $3 \mathrm{c}$ and $4 \mathrm{c}$ ). All features defined at the new ramp "N" $\left(\widetilde{B}, \theta_{B}\right.$ and $\phi_{B}$ variations and local ion dynamics) also apply to the old ramp
"O." From this steepened ramp "N," a large-amplitude whistler precursor starts to be emitted that leads to the next phase.

\subsection{Spiky SLAMS}

[18] As seen in Figure 2, the propagation of the SLAMS stops in the shock rest frame, at the time its steepened leading edge becomes a new shock front. Figure $3 \mathrm{~d}$ shows the profiles in the "spiky-SLAMS" phase at $\widetilde{t}=26.4 \widetilde{\omega}_{c i}^{-1}$. The spatial extent of the SLAMS is $\sim 13 \widetilde{c} / \widetilde{\omega}_{p i}$, and the shock ramp " $\mathrm{N}$ " is located at $\widetilde{x} \sim 11245$. 


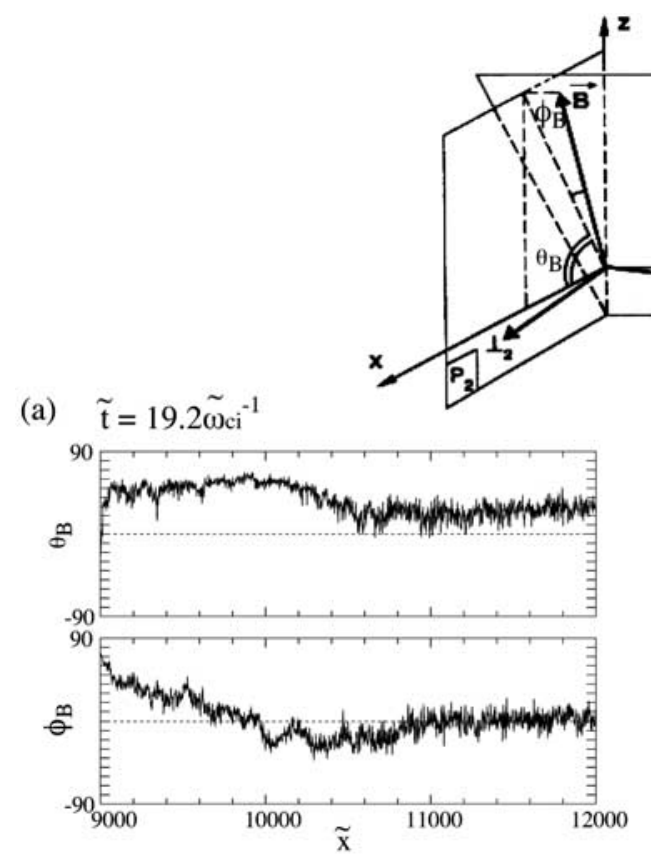

(b) $\tilde{\mathrm{t}}=23.6 \tilde{\omega}_{\mathrm{ci}}{ }^{-1}$

(c) $\tilde{t}=25.2 \tilde{\omega}_{\mathrm{ci}}^{-1}$

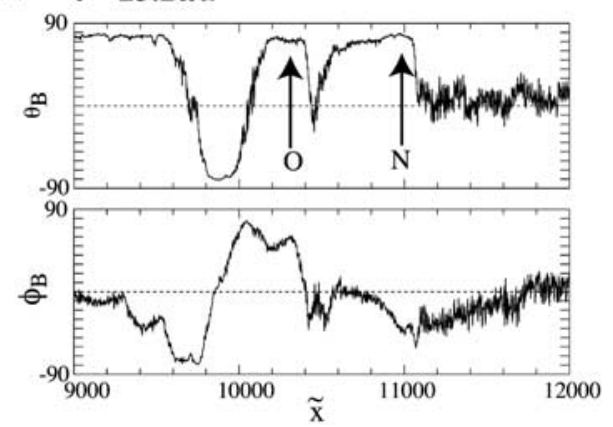

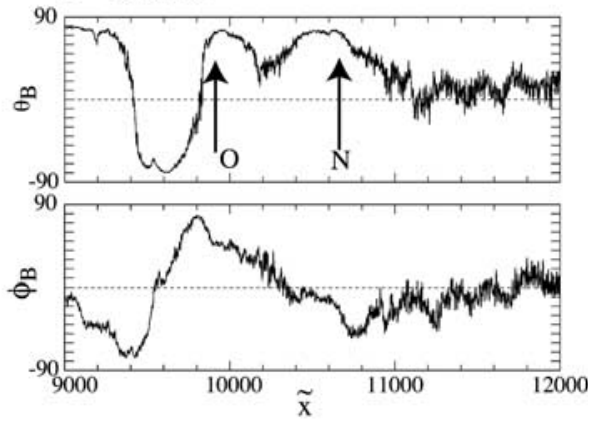

(d) $\tilde{\mathrm{t}}=26.4 \tilde{\omega}_{\mathrm{ci}}{ }^{-1}$

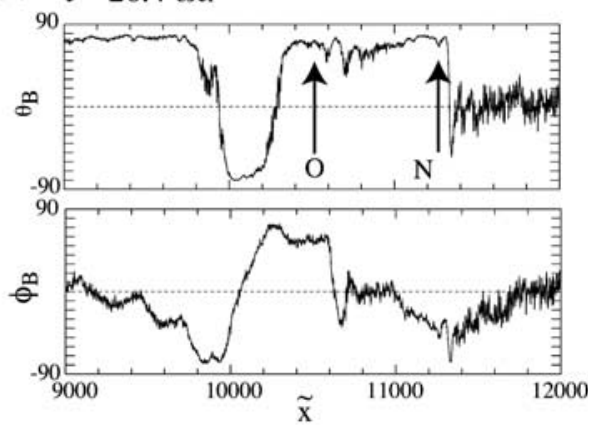

Figure 4. Same as Figure 3 for the rotation angles $\theta_{B}$ and $\phi_{B}$ of the self-consistent magnetic field. Angles $\theta_{B}$ and $\phi_{B}$ define the rotation of the self-consistent $\widetilde{B}$ field within and outside the coplanarity plane as represented by the sketch at the top of the figure. This plane is defined as containing the magnetostatic field and the normal ( $x$ axis) to the shock front.

[19] The whistler precursor is well separated from the new ramp " $\mathrm{N}$ " of the oblique (quasi-perpendicular) shock front with a characteristic length scale $\widetilde{\lambda}_{w, N} \approx 2-3 \widetilde{c} / \widetilde{\omega}_{p i}$ (Figure 3d). This emission forces the magnetic field to rotate locally towards negative $\theta_{B}\left(\approx-55^{\circ}\right)$ in front of the ramp "N"; in contrast, $\theta_{B}$ always takes large positive values approaching $90^{\circ}$ at "N" (Figure $4 \mathrm{~d}$ ). However, $\phi_{B}$ always stays negative through both the whistler and the new ramp " $\mathrm{N}$ " during the different phases of the SLAMS evolution. Figure 2 clearly shows that the whistler emission persists during a limited time range between $\widetilde{t}=25.8$ and $28.3 \widetilde{\omega}_{c i}^{-1}$. Starting at $\widetilde{t}=25.44 \widetilde{\omega}_{c i}^{-1}$, a similar whistler emission is also observed from the old shock ramp "O" with a wavelength $\widetilde{\lambda}_{w, O} \sim 2-3 \widetilde{c} / \widetilde{\omega}_{p i}$. As a consequence, the trailing edge of the SLAMS becomes more difficult to be distinguished from the old shock front ("O" at $\widetilde{x} \sim 10600$ ) and crashes into it. In addition, ions within the far region in the trailing edge of the SLAMS start to be heated by the propagating whistler emitted from the old ramp "O." In contrast with the previous phase, strong differences occur in the variations of $\theta_{B}$ and $\phi_{B}$ between the leading and the trailing edges of the SLAMS, but the $\theta_{B}$ angle shows little variation between the trailing edge of the SLAMS and the old ramp "O" (Figure 4d).

[20] The electrostatic field $\widetilde{E}_{l x}$ has now reached a noticeable value at the new shock front over an extent corresponding to the spatial width of the magnetic front including the ramp and the whistler precursor $\left(\widetilde{L}_{l e} \approx 200\right.$ $\Delta=4.7 \widetilde{c} / \widetilde{\omega}_{p i}$ in Figure 3d). However, one new result is that a very spiky pattern builds up in the electrostatic field $\widetilde{E}_{l x}$ over a narrow width $\widetilde{L}_{s} \approx 0.56 \widetilde{c} / \widetilde{\omega}_{p i} \approx 4 \widetilde{c} / \widetilde{\omega}_{p e}$ (within the front width $\widetilde{L}_{l e}$ ), which is much smaller than the width of the shock ramp "N" or the whistler wavelength. This feature is characteristic of the so-called "spiky-SLAMS" phase. The enlarged view of Figure 5 shows that the spiky $\widetilde{E}_{l x}$ amplitude at $\widetilde{x} \sim 11331$ is far beyond the background noise level. There are about 24 grids within this spiky $\widetilde{E}_{l x}$ so that the spatial resolution is high enough to confirm its full physical meaning. Moreover, one should notice that the spiky $\widetilde{E}_{l x}$ rises up within the ramp of the whistler itself and not at the 


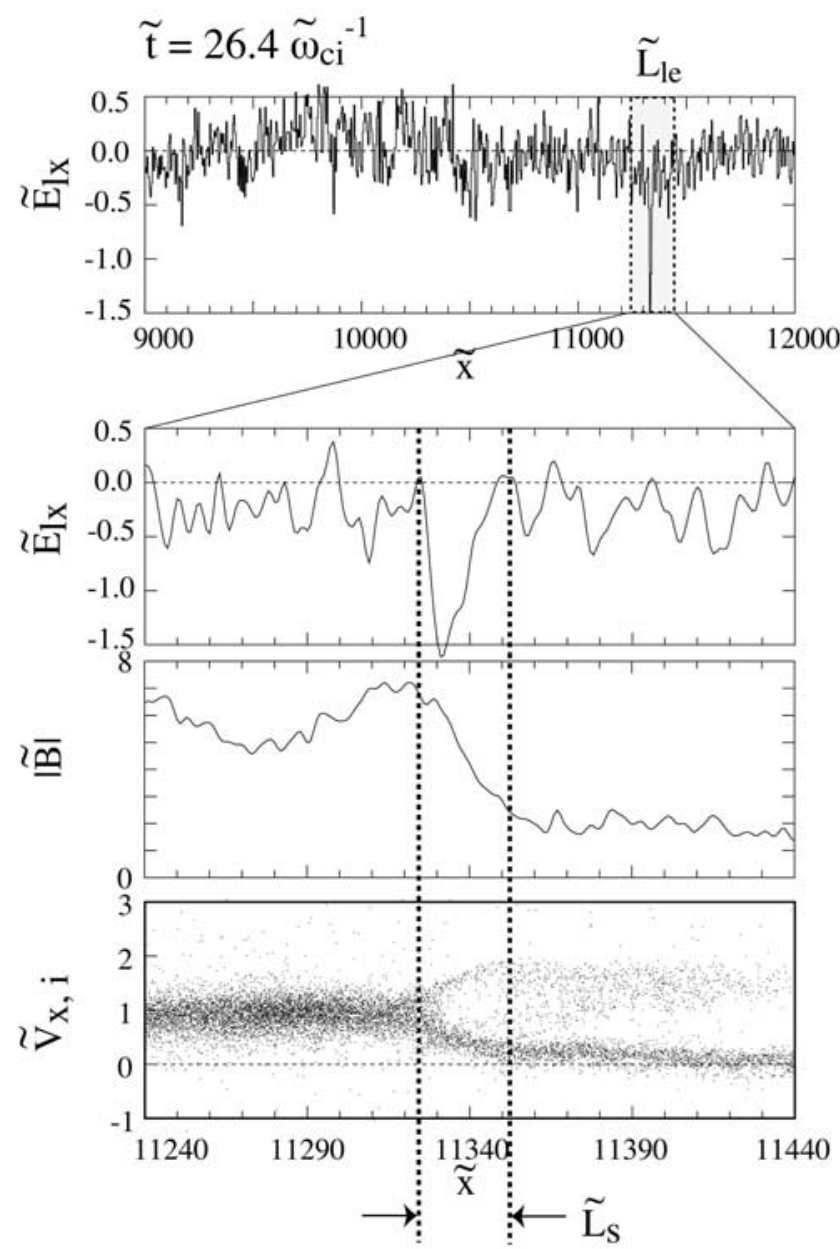

Figure 5. Enlarged plot of the electrostatic field $\widetilde{E}_{l x}$, the amplitude of the magnetic field $|\widetilde{B}|$, and the ion phase space $\left(\widetilde{v}_{x, i}, \widetilde{x}\right)$ at time $\widetilde{t}=26.4 \widetilde{\omega}_{c i}^{-1}$.

shock ramp "N." Indeed, the whistler precursor reaches a strong amplitude at least comparable to (or even larger than) that of the $\widetilde{B}$ field at the ramp "N," once it is emitted from the ramp "N." The location of this largest $\widetilde{B}$ field is associated with a noticeable ion trapping (well defined internal vortex) as seen in the ion phase space of Figure 5. This means that the whistler precursor propagates with a velocity appropriate to feed the ion trapping and reaches an amplitude large enough to act as a new shock ramp (located at $\widetilde{x}=11323 \Delta$ ), well ahead of the ramp "N" (located at $\widetilde{x}=$ $11245 \Delta$ ). Another important result is that this spiky $\widetilde{E}_{l x}$ field has an intermittent occurrence, since it only survives within a time range $\sim 0.8 \widetilde{\omega}_{c i}^{-1}$. However, it has a strong impact on the ion dynamics since it initiates a strong local and specular ion reflection. At this stage, the steepening process progressively stops because of the local balance brought by the dispersive effects and whistler waves are radiated upstream (Figure 2).

[21] In the present plasma conditions, results show that electron dynamics is relatively poor and only suffer some compression through the SLAMS. This is in strong contrast with ion dynamics (trapping) as shown in Figure 5. This difference is due to the fact that the whistler velocity $\widetilde{v}_{w, N}$ is much higher than the ion thermal velocity $\widetilde{v}_{t h, i}$ but of the order of the electron thermal velocity $\widetilde{v}_{t h, e}$ As a consequence, a large-amplitude electrostatic field $E_{l x}$ is necessary to drag the most energetic of the background ions to the resonant region $\left(\widetilde{v}_{w, N}\right)$ and to initiate trapping. This difference between ion and electron dynamics is at the origin of the local large amplitude space charge effects field $\widetilde{E}_{l x}$ (effects included self-consistently in the PIC code). The field amplitude may vary according to different upstream plasma conditions and various Mach regimes which can affect the local values $\widetilde{v}_{w, N} / \widetilde{v}_{t h, i}$ and $\widetilde{v}_{w, N} / \widetilde{v}_{t h, e}$, but its large amplitude value is expected to persist as long as the ratio $\widetilde{v}_{w, N} / \widetilde{v}_{t h, i}$ is high enough, and the dynamics of electrons and ions strongly differs.

\subsection{Late SLAMS}

[22] The fact that the front of the SLAMS is locally behaving as a local quasi-perpendicular shock strongly affects its internal dynamics and may delay its large scale reformation process in the "late SLAMS" phase $\widetilde{t}>$ $\left.26.4 \widetilde{\omega}_{c i}^{-1}\right)$. This phase is more difficult to analyze because of the simultaneous occurrence of local dispersion/dissipation processes. This leads the SLAMS to lose progressively its initial monolithic pattern and to the formation of some "sub-SLAMS" structures at later times. We will come back to this point later on.

[23] At the present stage, local ion reflection forces upstream ions to gyrate and to penetrate into the downstream region of the SLAMS front after the full gyration is completed. As a consequence, the field-aligned ion reflection and the feeding of more diffuse ions over a very large scale upstream are more difficult to establish; no ULF waves can spontaneously rise up. Local specular ion reflection is the dominant process. An important percentage of upstream ions are reflected against the new shock front and accumulate upstream to form a foot-like pattern with a width of the order of the trapping loop scale similar to the results of Lembège and Dawson [1987]. This foot is characteristic of a quasi-perpendicular shock in supercritical regime. Let us note that the whistler precursor emitted from the ramp "N" is well evidenced in the $\widetilde{E}_{l x}$ and $\widetilde{B}$ field profiles and may partially hide the foot signature. However, the foot is clearly apparent in the density profile and in the ion phase space. The foot formation is relatively rapid since it is directly related to the large amplitude of the local $\widetilde{B}$ field and the spiky $\widetilde{E}_{l x}$ (at the whistler location) so that a local $\widetilde{E}_{l x}$ starts to build up within the foot and to interact with reflected ions before these complete their full gyromotion and penetrate into the downstream region of the SLAMS (not shown here). The initially well-defined trapping loop vortex is progressively deformed upstream and blurs out. Indeed, a part of reflected ions lose partially their coherence during their gyromotion and become more diffuse. This situation is similar to that already discussed in Figure 4a of Lembège and Dawson [1987].

[24] At later times, this ion accumulation is large enough to initiate a self-reformation of the shock front. This cyclic process is identified by arrows in Figure 6. It is characterized by a cyclic period $\sim 384 \widetilde{\omega}_{p e}^{-1}=1.92 \widetilde{\omega}_{c i}^{-1}$ which is of the order of the mean ion cyclotron period measured around the overshoot $\left(\widetilde{\tau}_{c i}^{\text {overshoot }}=350 \widetilde{\omega}_{p e}^{-1}\right)$ as already observed for a quasi-perpendicular shock by Lembège and Dawson [1987] and Lembège and Savoini [1992] in 1-D and 2-D 


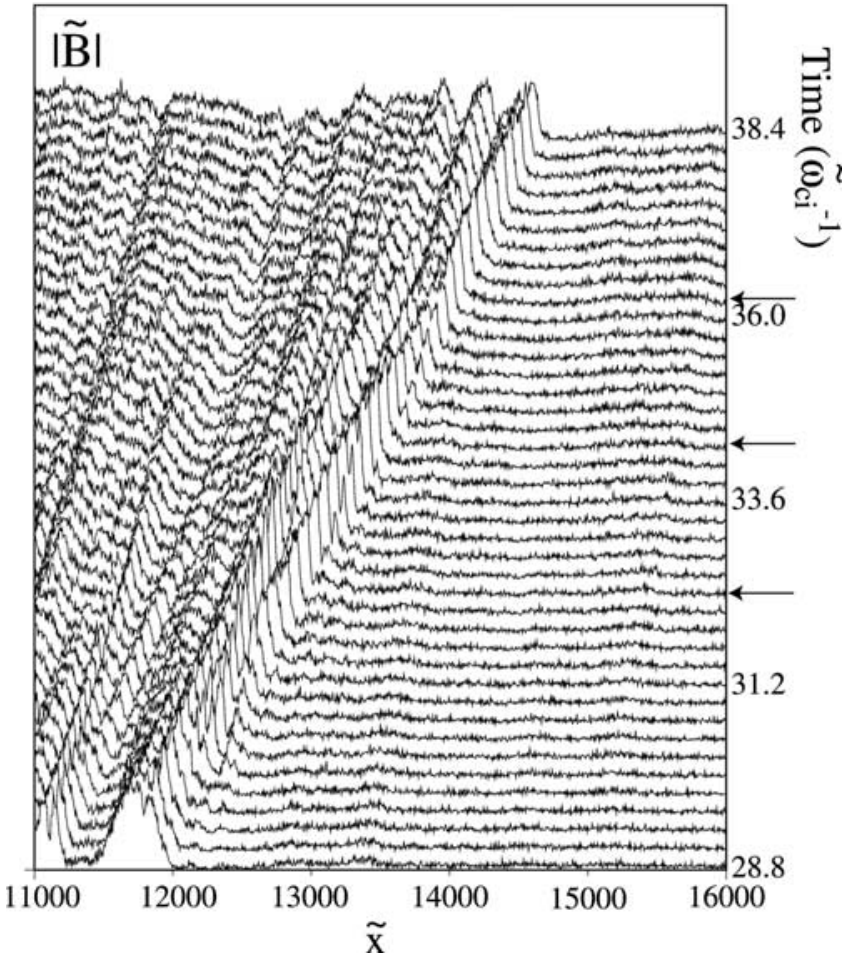

Figure 6. Time stackplot of the magnetic field amplitude profile $|\widetilde{B}|$ in the "late SLAMS" phase $\left(\widetilde{t}>26.4 \widetilde{\omega}_{c i}^{-1}\right)$. Arrows indicate the cyclic reformation of the SLAMS leading edge.

full particle simulations, respectively. This self-reformation dynamics is very local in space and establishes over spatial/ time scales much smaller than the very large scales intrinsic to the other self-reformation processes of quasi-parallel shocks.

[25] After a few subcycles of reformation $\left.\widetilde{t}>36.24 \widetilde{\omega}_{c i}^{-1}\right)$, no self-reformation occurs (Figure 6), although some ion reflection still persists. One invoked reason is that different ion populations (background and diffuse ions) are now present upstream of the SLAMS leading edge, and can increase the local upstream $\beta_{i}$ parameter (ratio of the ion thermal energy over the magnetic energy). Simultaneously, the velocity $\widetilde{v}_{l e}$ of the SLAMS leading edge is slightly decreasing in time (Figure 7). Even if some ions are still reflected, these two changes lead to a decrease of the ratio $\widetilde{v}_{l e} \widetilde{v}_{t h, i}$, which affects the way how reflected ions accumulate in front of the SLAMS. When this ratio is only a few units, reflected ions lose some coherence during their gyromotion (no clear trapping loop), become more diffuse and accumulate over a wide extent which is hidden within the width of the SLAMS front. Foot and ramp become mixed and cannot be distinguished each other as shown by recent simulations [Hada et al., 2003; Scholer et al., 2003a]. In these conditions, any local self-reformation of quasiperpendicular shock front dies out.

[26] Figure 6 shows how the initial SLAMS progressively degenerates during the "late-SLAMS" phase in that its initial width increases in time and substructures occur in its downstream region (behind ramp "N"). This spatial broadening is due to the fact the leading edge of the whistler precursor is propagating with velocity higher than the ramp "N." A very long computer run should be necessary in order to recover the formation of a new SLAMS directly related to the cyclic self-reformation of the quasi-parallel shock. In the present case, the simulation run has been stopped before any new SLAMS clearly builds up. However, these results stress that a certain care is necessary when identifying any SLAMS pattern. One important parameter is the SLAMS life time which needs to be large enough in order to be identified during a satellite crossing. The question whether these substructures can be considered as smaller scale SLAMS (isolated or/and embedded) will be analyzed in a separated study.

\section{Comparison With Previous Results and Discussion}

\subsection{Comparison of PIC and Hybrid Simulation Results}

[27] For a better comparison, the present plasma conditions have been chosen in order to approach those used in previous hybrid simulations $\left(\theta_{B o}=30^{\circ}\right.$ and $\left.\beta_{i, e}=0.5\right)$, except $M_{A}=5.8$ (our run) and 4.6 (Paper 1). Present results confirm main results issued from Paper 1, which reinforce their validation. First, SLAMS may originate from ULF waves which are excited upstream of the shock front by the interaction of reflected diffuse ions with background ion plasma. In other words, SLAMS and ULF upstream waves are the same entity at different stages of their formation. In our case, the once formed monolithic $\widetilde{B}$ field profile is well detached from the "old" shock front and is identified as an isolated-type SLAMS. It stays almost stationary with respect to the old front. At later time, the leading edge of the SLAMS strongly steepens, becomes a new shock front, and progressively loses its monolithic pattern during its spatial expansion. Second, characteristic scales deduced from results of Paper 1 are summarized as follows: the SLAMS extent varies from $\approx 20 \widetilde{c} / \widetilde{\omega}_{p i}$ to $\approx 8 \widetilde{c} / \widetilde{\omega}_{p i}$ after shrinking, the magnetic field presents a jump of the order of $|\Delta \widetilde{B}| /\left|\widetilde{B}_{o}\right| \approx 2.2$ and rotates through the leading edge so that

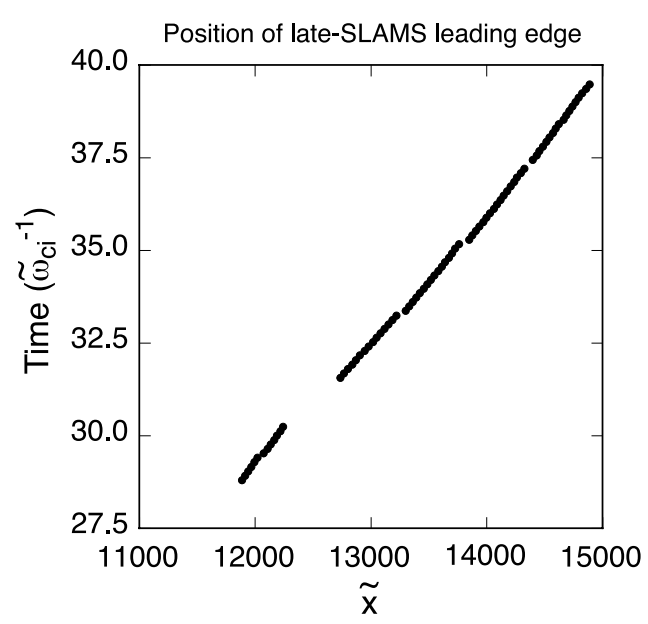

Figure 7. Location of the ion trapping loop measured at the leading edge of the SLAMS versus time during the "late SLAMS" phase. The slope of the curve provides the velocity $\widetilde{v}_{l e}$ of the leading edge. 
Table 2. Spatial and Timescales of ULF Waves, SLAMS, and Whistler Precursors Measured in the Present Full Particle Simulation $^{\mathrm{a}}$

\begin{tabular}{llll}
\hline \hline & $\begin{array}{c}\text { Symmetric } \\
\text { SLAMS }\end{array}$ & $\begin{array}{c}\text { Asymmetric } \\
\text { SLAMS }\end{array}$ & \multicolumn{1}{c}{$\begin{array}{c}\text { Spiky } \\
\text { SLAMS }\end{array}$} \\
\hline $\begin{array}{l}\text { SLAMS } \\
\text { Spatial extent }\end{array}$ & $14 \widetilde{c} / \widetilde{\omega}_{p i}$ & $14.2 \widetilde{c} / \widetilde{\omega}_{p i}$ & $13.2 \widetilde{c} / \widetilde{\omega}_{p i}$ \\
$\begin{array}{l}\text { Leading edge: } \\
\text { Ramp width }\end{array}$ & $6 \widetilde{c} / \widetilde{\omega}_{p i}$ & $2.47 \widetilde{c} / \widetilde{\omega}_{p i}$ & NA \\
Velocity & $4.47 \widetilde{v}_{A}$ & $4.8 \widetilde{v}_{A}$ & $5.25 \widetilde{v}_{A}$ \\
$\Delta \widetilde{B} / \widetilde{B}_{o}$ & 2.66 & 4.4 & 3.7 \\
Spiky $E_{l x}$ extent & NA & NA & $0.56 \widetilde{c} / \widetilde{\omega}_{p i}$ \\
Trailing edge: & & & \\
Ramp width & $3.54 \widetilde{c} / \widetilde{\omega}_{p i}$ & $5.11 \widetilde{c} / \widetilde{\omega}_{p i}$ & $6 \widetilde{c} / \widetilde{\omega}_{p i}$ \\
$\Delta \widetilde{B} / \widetilde{B}_{o}$ & 2.3 & 1.6 & 1.3 \\
\hline & "ULF wave growth" phase & \\
\hline
\end{tabular}

ULF wave

Wavelength $\tilde{\lambda} / 2 \quad 35 \widetilde{c} / \widetilde{\omega}_{p i}$

Velocity $\quad 3 \widetilde{v}_{A}$

Frequency $\quad 0.5 \widetilde{\omega}_{c i}$

"Spiky-SLAMS"phase

Whistler precursor

\begin{tabular}{lll} 
& $\begin{array}{l}\text { From new } \\
\text { ramp "N" }\end{array}$ & $\begin{array}{l}\text { From the old } \\
\text { ramp "O" }\end{array}$ \\
Wavelength & $2.5 \widetilde{c} / \widetilde{\omega}_{p i}$ & $2.7 \widetilde{c} / \widetilde{\omega}_{p i}$ \\
Velocity & $5.6 \widetilde{v}_{A}$ & $3 \widetilde{v}_{A}$ \\
Frequency & $13.9 \widetilde{\omega}_{c i}$ & $6.8 \widetilde{\omega}_{c i}$ \\
\hline
\end{tabular}

a"NA" holds for "not available."

it approaches the features of a local quasi-perpendicular shock, and a whistler precursor is emitted both from the ramp of the old shock and from the SLAMS leading edge with a wavelength $\approx 3.3 \widetilde{c} / \widetilde{\omega}_{p i}$. These scales are in a good agreement with values of Table 2 . In the present case, the whistler emission from " $\mathrm{N}$ " and "O" ramps starts (at $\widetilde{t}=$ $25.8 \widetilde{\omega}_{c i}^{-1}$ and $25.44 \widetilde{\omega}_{c i}^{-1}$, respectively) shortly after the ULF transforms into the SLAMS, i.e., no ions are fully reflected specularly yet. This suggests that whistler precursor is mainly due to dispersive effects and is not related to some reflected ion/upstream ion instability. This result is similar to conclusions of Paper 1.

[28] However, strong differences appear between both types of simulations and are summarized as follows:

[29] 1. One key advantage of PIC simulations is the accessibility to time/spatial scales much lower than the ion scales, even if electron dynamics does not play a crucial role as in the present case; electrons only suffer some compression heating. This accessibility allows (1) a highresolution analysis of the large amplitude whistler precursor emitted both from the old ("O") and the new ("N") ramp, (2) the build-up of a spiky electrostatic field over a spatial scale much smaller than one ion inertia length, (3) this spiky field $\widetilde{E}_{l x}$ to rise up within the large-amplitude whistler once emitted from the leading edge of the SLAMS and to persist over a limited time less than one $\widetilde{\omega}_{c i}^{-1}$, and (4) a cyclic selfreformation of the SLAMS front behaving as a local supercritical quasi-perpendicular shock. All these features could not be evidenced in Paper 1 and in other previous hybrid simulations. In particular, the local self-reformation (point 4) which allows to maintain the SLAMS pattern during a noticeable time range could not take place since the spatial resolution was too low as shown by Hellinger et al. [2002] for a perpendicular shock.
[30] 2. As shown in Paper 1, one condition for upstream waves to grow and to shrink until forming SLAMS is that the gradient scale of the diffuse ion density is comparable to the original ULF wavelength. In Paper 1, the Maxwellian distribution in the upstream system is artificially divided into two parts in initial conditions: a core part (with low temperature) and an outer part (with high temperature). Each macro-ion from the outer part is split into six new ions with the same velocity components; these ions are placed at random in the same cell at slightly different positions. This procedure allows to represent backstreaming diffuse ions (hot part) with sufficient statistics. However, the mechanisms responsible for this "reinforced" ion diffusion are unknown. In addition, this procedure can speed up artificially some mechanisms (i.e., ULF waves shrinking), with respect to other mechanisms (whistler precursor emission for instance); as a consequence the relative contribution of different mechanisms to the intrinsic dynamics of the quasi-parallel shock may be affected. In contrast, in our present case, one Maxwellian ion population is introduced in initial conditions so that diffuse ions are progressively formed by self-consistent interactions with waves at times before the ULF wave builds up $\left(\widetilde{t}<19.2 \widetilde{\omega}_{c i}^{-1}\right)$. The mechanisms responsible for the formation of diffuse ions are out of scope in the present paper and will be analyzed in a further study. As a consequence, diffuse ions are well present in the upstream region where the ULF wave starts to grow, while partially diffuse ions (shell distribution) are present far upstream from this region (Figure 3a). Such conditions reveal to be sufficient to initiate the growth and the shrinking of the ULF wave at later times $\left.\widetilde{(t}>19.2 \widetilde{\omega}_{c i}^{-1}\right)$ without any numerical artifact. However, in the present case only one wavelength SLAMS has been analyzed, which allows to follow its full dynamics ("growth" until "decay") before any large-scale self-reformation of the quasi-parallel shock takes place. In contrast, in Paper 1, diffuse ions are "rapidly" present even far from the initial shock front, which allows the ULF wave to grow over several wavelengths (wave development indicated by indexes 1 and 2 in Figure 3 of Paper 1) and to reinitiate shortly a new cyclic self-reformation immediately after the formation of the first SLAMS (initially named wave 1 in Paper 1).

[31] 3. In the present case, the dynamics of the whistler precursor plays an important role as a new shock front during and after the "spiky-SLAMS" phase. It is strongly related to the local formation of specularly reflected ions, in contrast with Paper 1. This means that the SLAMS dynamics at late time strongly depends on the relative occurrence of the whistler precursor with respect to the ULF wave growth, i.e., to the set up of a strong density gradient of diffuse ions upstream. These characteristic occurrence times vary relatively with the upstream plasma conditions and the Mach regime of concern. These differences suggest that no unique mechanism participates to the overall dynamics of SLAMS after it builds up, but our present knowledge indicate that the build up process of the SLAMS from ULF waves is unique.

\subsection{Comparison With Experimental Results}

[32] Our results have been also compared with previous experimental observations. As a reference, the statistics obtained by Schwartz et al. [1992] and Mann et al. [1994] are summarized in Table 3, which shows typical 
Table 3. Comparison of the Typical Scales of the SLAMS Between the PIC Simulations (Present Results) and Observations [Schwartz et al., 1992; Mann et al., 1994] ${ }^{\mathrm{a}}$

\begin{tabular}{lcccccc}
\hline & Time & Spatial Width & $\left|B_{\max }-B_{o}\right| /\left|B_{o}\right|$ & Upstream $M_{A}$ & $\beta$ & $\theta_{B o}$ \\
\hline PIC simulations & $5-6 \widetilde{\omega}_{c i}^{-1}$ & $13-15 \widetilde{c} / \widetilde{\omega}_{p i}$ & $2.6-4.4$ & 5.8 & 1.0 & $30^{\circ}$ \\
Observations & $2-10 \widetilde{\omega}_{c i}^{-1}$ & $10-70 \widetilde{c} / \widetilde{\omega}_{p i}$ & $2.72 \pm 1.17$ & 7.5 & $5-6$ & $32.3 \pm 14.5^{\circ}$ \\
\hline
\end{tabular}

${ }^{\mathrm{a}}$ Reference scales for observations are $\widetilde{c} / \widetilde{\omega}_{p i} \approx 91 \mathrm{~km}$ and $\widetilde{\omega}_{c i}^{-1} \approx 2.1 \mathrm{~s}$.

parameters characterizing SLAMS. The ranges on $\theta_{B o}$ are almost comparable between our results and the referred observations. In these references, the value of plasma beta was relatively high $(5-6)$, in contrast with present conditions where $\beta \sim 1$. One expected consequence of this difference is that self-reformation of the SLAMS front mentioned in the late-SLAMS phase (section 3.6) may have more difficulties to establish for higher ion $\beta_{i}$, according to the results of Hada et al. [2003] and of Scholer et al. [2003a]. Moreover, with typical solar wind parameters, $\omega_{c i}^{-1} \sim 2.1$ seconds and $c / \omega_{p i} \sim 91 \mathrm{~km}$, spatial and temporal scales of SLAMS issued from our simulation results are in good agreement with observations. Moreover, whistler-like wave trains attached to the SLAMS edges were often observed experimentally, which we have identified in two ways. One is emitted from the "new" shock ramp ("N") before the spiky electrostatic field appears; the other one is emitted from the old shock ramp ("O") which overcomes the trailing part of the newly formed SLAMS.

[33] Recently, Lucek et al. [2002] have identified SLAMS-like structures from the magnetic field data of Cluster-2 and showed different signatures between spacecrafts only $\sim 400-1000 \mathrm{~km}$ of Cluster-2 tetrahedron scales apart. Upstream plasma conditions (Mach number $M_{A}=$ 12-13) were different from the present numerical conditions. However, these experimental observations stress several features of SLAMS which are well retrieved in the present results: (1) rotation of the magnetic field approaching $90^{\circ}$ through the leading edge of the SLAMS, (2) the leading edge of the SLAMS is acting as a local quasiperpendicular shock in supercritical regime with the formation of a foot (however, the foot-like pattern was evidenced in observations by the magnetic field signature only, because no corresponding plasma data have been presented), (3) emission of high frequency whistler associated with the leading edge, (4) right-handed polarization of the B field through the leading edge propagating sunward, (5) the variation of the $\mathrm{B}$ field orientation is different through the leading and the trailing edge of the SLAMS (which corresponds to a late time of the "asymmetric-SLAMS" phase), and (6) formation of a plateau in the rear part of the SLAMS profile (similar to Figure 3c). It confirms also that the steepened ULF waves typically have a scale much larger than the spacecraft separation $(\sim 400-1000 \mathrm{~km}$, with an upstream ion inertia length $\sim 135 \mathrm{~km}$ ). One has to notice that our simulations are limited to one dimension which assumes that the extent of the crossed SLAMS is large enough to be considered as a planar structure.

[34] Present results stress that different SLAMS signatures by multisatellites mission Cluster-2 may result from the crossing of the same entity at different stages of its evolution. In contrast, Cluster-2 observations suggest that these structures are not planar on the scale of the tetrahedron and these are three-dimensional entities. However, one still ignores whether the different signatures observed between the four satellites are the consequences of the three dimensionality or of a rugged leading edge of the SLAMS (with a rippling scale smaller than or comparable to the spacecraft separation). At this stage, present simulations allow to analyze small scale structures which are expected within SLAMS along the shock normal (for instance, the spiky electrostatic field and/or high-resolution whistler precursor) and which can be compared with observations, provided that the spacecrafts separation is appropriate. This separation can be estimated from the spatial scales measured at different times of the ULF waves growth and of the SLAMS development. Present results and Table 2 provide such scales normalized to the upstream ion inertial length so that these can be used for different plasma conditions where $\widetilde{c} / \widetilde{\omega}_{p i}$ has changed. For instance, let us consider SLAMS in the shock rest frame. The spatial structure analysis of a same SLAMS (during the asymmetric SLAMS or spiky SLAMS phases when its extent is the smallest) crossed by two successive spacecrafts approximately aligned along the shock normal, requires a spacecrafts separation $L_{s a t} \approx$ $1300-1400 \mathrm{~km}$, assuming $\widetilde{c} / \widetilde{\omega}_{p i} \sim 100 \mathrm{~km}$. Moreover, a precise analysis of the SLAMS leading edge by these two same spacecrafts will require a smaller separation $L_{\text {sat }} \approx$ $200-300 \mathrm{~km}$. In contrast, the analysis of the same entity (ULF waves versus SLAMS pattern) will require a much larger spacecrafts separation.

[35] Moreover, recent experimental electric field measurements of SLAMS have been performed on board of Cluster-2 by Behlke et al. [2003]. These observations confirm quite well local ion trapping and evidence an electric field signature very similar to the spiky electric field measured at the leading edge of the SLAMS in the present results (Figures 3d and 5). However, the scale of the observed electric field (of the order of electron gyroradius) is smaller than that found in the present simulations. This difference could be explained by the fact that the Mach regime measured experimentally $(\sim 12-13)$ is higher than in the present simulations, which can affect the gradient of the electrostatic field. The similarity in the spiky electric field between observations and numerical results is a quite stimulating feature, which requires further quantitative analysis. On one hand, complementary observations are needed in order to confirm this feature for different SLAMS crossings. This will require an important statistical analysis because of the intermittent nature of this spiky pattern. On the other hand, the amplitude variations of the spiky $E_{l x}$ field versus numerical parameters (such as mass ratio and/or the ratio $\omega_{p e} / \omega_{c e}$ ) require parametric analysis which are left for a further work.

\section{Conclusions}

[36] The main advantage of full particle treatment is to analyze the SLAMS dynamics with resolution much higher 
than that used in previous studies in particular over scales much smaller than ion scales $\left(\widetilde{c} / \widetilde{\omega}_{p i}, \widetilde{\omega}_{c i}^{-1}\right)$. The understanding of SLAMS time evolution is important for various reasons: (1) monolithic patterns characterizing SLAMS have been currently observed by different spacecrafts missions which strongly suggests that these are "robust" signatures intrinsic to the quasi-parallel shocks dynamics, and (2) observations of SLAMS within regions of ULF waves activity during a same quasi-parallel shock crossing suggest that both SLAMS and ULF waves play a key role in the dynamics of a quasi-parallel shock. Precisely, these measurements suggest that SLAMS appear to grow out of the ULF wave field [Thomsen et al., 1990; Schwartz, 1991; Schwartz et al., 1992]. Presently, successive phases of the SLAMS dynamics have been defined in order to identify carefully the spatial changes that SLAMS suffer, and the time ranges over which these changes take place from the growth of ULF waves. Time and spatial scales measured in each phase allow to prepare a further comparison with experimental measurements and to provide estimates of spacecrafts separation for Cluster-2 mission too. These values are in good agreement with results already obtained with hybrid simulations of Paper 1 , which confirms their validation. In addition, these results clearly confirm that upstream ULF waves and SLAMS are signatures of the same entity measured at different times of their evolution. These also show how the steepened leading edge of the SLAMS evolves in time and transforms into a local quasiperpendicular shock front. A certain percentage of upstream background ions are specularly reflected (trapping) at this new shock front, and form a local foot. Moreover, the accessibility to electron scales allows to evidence the following new results: (1) the whistler precursor emitted both from the old ("O") shock ramp and the new ("N") ramp (i.e., the leading edge of the SLAMS) has been analyzed with high resolution; results show that it strongly steepens until reaching a large amplitude comparable to that at the ramp; (2) a spiky electrostatic field $\widetilde{E}_{l x}$ builds up over a narrow width much smaller than the steepened ramp, within the large amplitude whistler emitted from the leading edge, (3) this spiky $\widetilde{E}_{l x}$ field has a limited life time less than one $\widetilde{\omega}_{c i}^{-1}$; this intermittent occurrence can be used as a reference signature in the time history of the SLAMS dynamics and/or as the end of a cyclic self-reformation of the quasi-parallel shock, (4) at later times, this local spiky whistler acts as a new shock ramp with a width smaller than one $\widetilde{c} / \widetilde{\omega}_{p i}$, and (5) the accumulation of reflected ions (foot build-up) is strong enough to initiate a local self-reformation of the SLAMS front similar to that commonly observed for a quasi-perpendicular shock in supercritical regime. All these fine scale results could not be observed in previous hybrid simulations because of a too low spatial resolution. During the "late SLAMS" phase, the SLAMS are spreading spatially and progressively lose its initial monolithic feature, giving birth to some substructures. Present results are also in good agreement with typical scales of SLAMS patterns evidenced in experimental results of AMPTE UKS/IRM satellites, and more recently of the Cluster-2 multisatellite mission.

[37] Let us note that during the revision period of the present paper, other results of full particle simulations of SLAMS and quasi-parallel shocks have been published by
Scholer et al. [2003b]. These recent simulations differ from the present analysis in that these are not dedicated to the dynamics of SLAMS (although these have been also evidenced) but rather to the respective contribution of SLAMS (emerging from the growth of ULF waves) and of the phase standing whistler precursor emitted from the steepened edge of the SLAMS, in the self-reformation process of the quasi-parallel shock. The authors have essentially recovered both features described on the basis of full particle simulations in the short simulation system by Pantellini et al. [1992] and on the basis of hybrid simulations in a large simulation system by Scholer [1993]. These final results confirm that the large-scale self-reformation process of quasi-parallel shocks is mainly due to the ULF waves and associated SLAMS. These results will be compared in details with our simulations in a further analysis.

[38] Present results complete the sketch initially proposed by Schwartz and Burgess [1991] and present the quasiparallel shock as a patchwork of structures (enhancements of the magnetic field) at different times of their evolution including long-wavelength ULF waves, monolithic-type signatures symmetric in leading and trailing edges or strongly asymmetric (so that the leading edge presents all features of a quasi-perpendicular shock), and substructures of smaller scales (corresponding to reminiscent tracks of "old" monolithic SLAMS). An extension of the present analysis consists in analyzing the mechanisms responsible for the formation of diffuse ions which are an important ingredient in the formation of ULF waves and resulting SLAMS and contribute to the self-reformation of the quasiparallel shock at later times.

[39] Acknowledgments. Simulation runs have been performed on the IBM SP3 supercomputer at IDRIS center at Orsay. The authors acknowledge the computing assistance of N. Grimas (IDRIS center). The work of K. T. is supported by the JSPS fellowships abroad.

[40] Shadia Rifia Habbal thanks Elizabeth A. Lucek and another referee for their assistance in evaluating this paper.

\section{References}

Behlke, R., M. André, S. C. Buchert, A. Vaivads, and A. Eriksson (2003), Multi-point electric field measurements of Short Large-Amplitude Magnetic Structures (SLAMS) at the Earth's quasi-parallel bow shock, Geophys. Res. Lett., 30(4), 1177, doi:10.1029/2002GL015871.

Burgess, D. (1989), Cyclic behavior at quasi-parallel collisionless shocks, Geophys. Res. Lett., 16, 345-348.

Dubouloz, N., and M. Scholer (1993), On the origin of short large amplitude magnetic structures upstream of quasi-parallel collisionless shocks, Geophys. Res. Lett., 20, 547.

Dubouloz, N., and M. Scholer (1995), Two-dimensional simulations of magnetic pulsations upstream of the Earth's bow shock, J. Geophys. Res., 100, 9461-9474.

Gary, S. P., C. W. Smith, M. A. Lee, M. L. Goldstein, and D. W. Forslund (1984), Electromagnetic ion beam instabilities, Phys. Fluids, 27, 1852 .

Giacalone, J., S. J. Schwartz, and D. Burgess (1993), Observations of suprathermal ions in association with SLAMS, Geophys. Res., 149152.

Giacalone, J., S. J. Schwartz, and D. Burgess (1994), Artificial spacecraft in hybrid simulations of the quasi-parallel Earth's bow shock: Analysis of time series versus spatial profiles and a separation strategy for Cluster, Ann. Geophys., 12, 591-601.

Hada, T., M. Oonishi, B. Lembège, and P. Savoini (2003), Shock front nonstationarity of supercritical perpendicular shocks, J. Geophys. Res., 108(A6), 1233, doi:10.1029/2002JA009339.

Hellinger, P., P. Travnicek, and H. Matsumoto (2002), Reformation of perpendicular shocks: hybrid simulations, Geophys. Res. Lett., 29(24), 2234, doi:10.1029/2002GL015915. 
Lembège, B. (2003), Full particle electromagnetic simulation of collisionless shocks, in Space Plasma Simulation, edited by J. Buchner, C. T. Dum, and M. Scholer, p. 54, Springer-Verlag, New York.

Lembège, B., and J. M. Dawson (1987), Self-consistent study of a perpendicular collisionless and nonresistive shock, Phys. Fluids, 30, 1767 1788

Lembège, B., and P. Savoini (1992), Nonstationarity of a two-dimensional quasiperpendicular supercritical collisionless shock by self-reformation, Phys. Fluids, 4, 3533-3548

Lucek, E. A., T. S. Horbury, M. W. Dunlop, P. J. Cargill, S. J. Schwartz A. Balogh, P. Brown, C. Carr, K. H. Fanacon, and E. Georgescu (2002), Cluster magnetic field observations at a quasi-parallel bow shock, Ann. Geophys., 20, 1699-1710.

Mann, G., H. Lühr, and W. Baumjohann (1994), Statistical analysis of short large-amplitude magnetic field structures in the vicinity of the quasiparallel bow shock, J. Geophys. Res., 99, 13,315-13,323.

Pantellini, F. G. E., A. Heron, J. C. Adam, and A. Mangeney (1992), The role of the whistler precursor during the cyclic reformation of a quasiparallel shock, J. Geophys. Res., 97, 1303-1311.

Savoini, P., and B. Lembège (1994), Electron dynamics in two- and onedimensional oblique supercritical collisionless magnetosonic shocks, J. Geophys. Res., 99, 6609-6635.

Scholer, M. (1993), Upstream waves, shocklets, short large-amplitude magnetic structures and the cyclic behavior of oblique quasi-paralle collisionless shocks, J. Geophys. Res., 98, 47-57.
Scholer, M., I. Shinohara, and S. Matsukiyo (2003a), Quasi-perpendicular shocks: Length scale of the cross-shock potential, shock reformation, and implication for shock surfing, J. Geophys. Res., 108(A1), 1014, doi:10.1029/2002JA009515.

Scholer, M., H. Kucharek, and I. Shinohara (2003b), Short large-amplitude magnetic structures and whistler wave precursors in a full-particle quasiparallel shock simulation, J. Geophys. Res., 108(A7), 1273, doi:10.1029/ 2002JA009820.

Schwartz, S. J. (1991), Magnetic field structures and related phenomena at quasi-parallel shocks, Adv. Space Res., 11(9), 231.

Schwartz, S. J., and D. Burgess (1991), Quasi-parallel shocks: A patchwork of three dimensional isolated structures, Geophys. Res. Lett., 18, 373376.

Schwartz, S. J., D. Burgess, W. P. Wilkinson, R. Kessel, M. Dunlop, and H. Lühr (1992), Observations of short-large amplitude magnetic structures at a quasi-parallel shock, J. Geophys. Res., 97, 4209-4227.

Thomsen, M. F., J. T. Gosling, S. J. Bame, T. G. Onsager, and C. T. Russell (1990), Two-state ion heating at quasi-parallel shocks, J. Geophys. Res, 95,6363 .

B. Lembège, CETP/IPSL/CNRS, 10-12 Avenue de l'Europe, F-78140 Velizy, France. (bertrand.lembege@cetp.ipsl.fr)

K. Tsubouchi, Radio Science Center for Space and Atmosphere, Kyoto University, Uji, 611-0011 Kyoto, Japan. (tsubo@kurasc.kyoto-u.ac.jp) 